\title{
Animal magic is helping patients enjoy their trip to the dentist
}

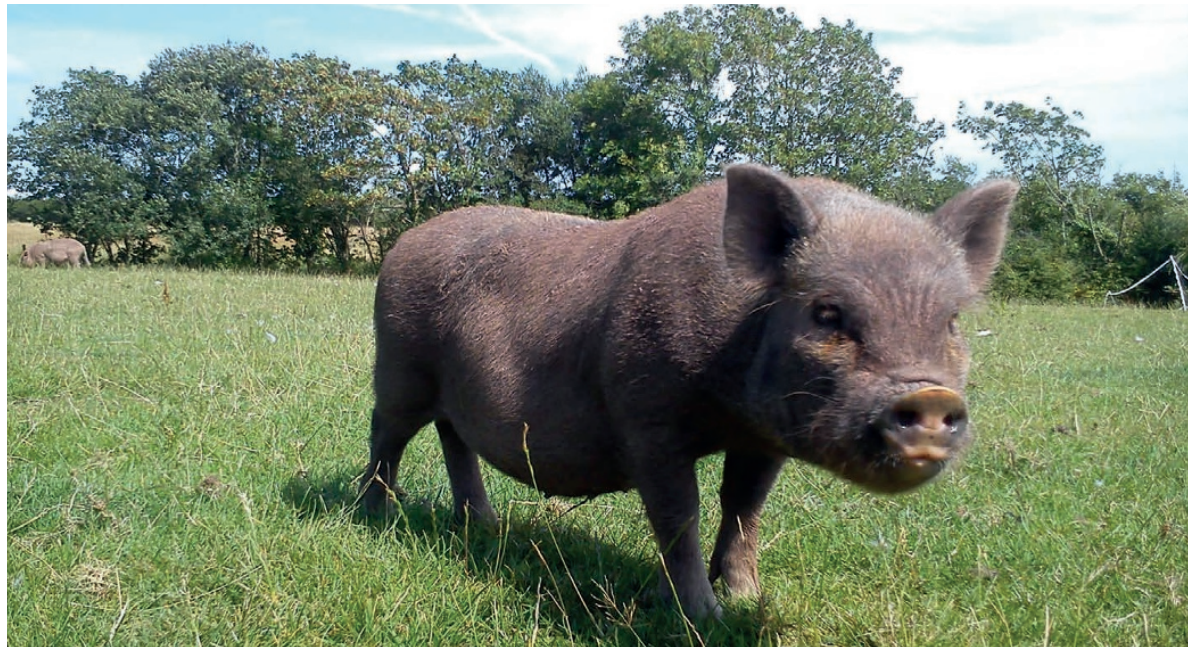

$\mathrm{P}$ atients nervous of visiting the dentist are being helped to overcome their phobias with a touch of 'animal magic' at an unusual dental practice in West Wales.

Various animals live in the grounds of the Herbrandston Dental Health Practice in Pembrokeshire and patients are invited and welcome to meet them while visiting for a dental appointment in the hope that this will relax them before treatment and build trust with the dental team.

The animals include two horses, five pigs, six emus, two ferrets, goats, chickens, ducks and turtles as well as sausage dogs.

The man behind the idea is principal dental surgeon Dr Mark Boulcott (pictured) who set up his private practice upon leaving the Armed Forces in 2004 and has used the land that came with the rural setting to house various animals.

'Because I am a sedationist as well, I'm interested in the psychology of people and dentistry in general,' said Boulcott. 'I wanted to do something that was different to attract people.

'We started attracting people who had phobias, and with children, I asked them if they and their parents wanted to come and meet the animals. You change their perception of coming to a dentist, and they're usually very obliging and you develop trust with them. It's modifying their behaviour and the way they look at dentists and dentistry in a different environment with different actions and it works very well.'

At another of Dr Boulcott's practices in Narbeth, he has patients who request that when they are recovering from sedation, they can have one of the practice's sausage dogs placed in their lap.

'We also offer this to adults', he added. 'If you're worried, particularly a young adult who has nobody coming with them and they have a fear or anxiety, they can feel overwhelmed

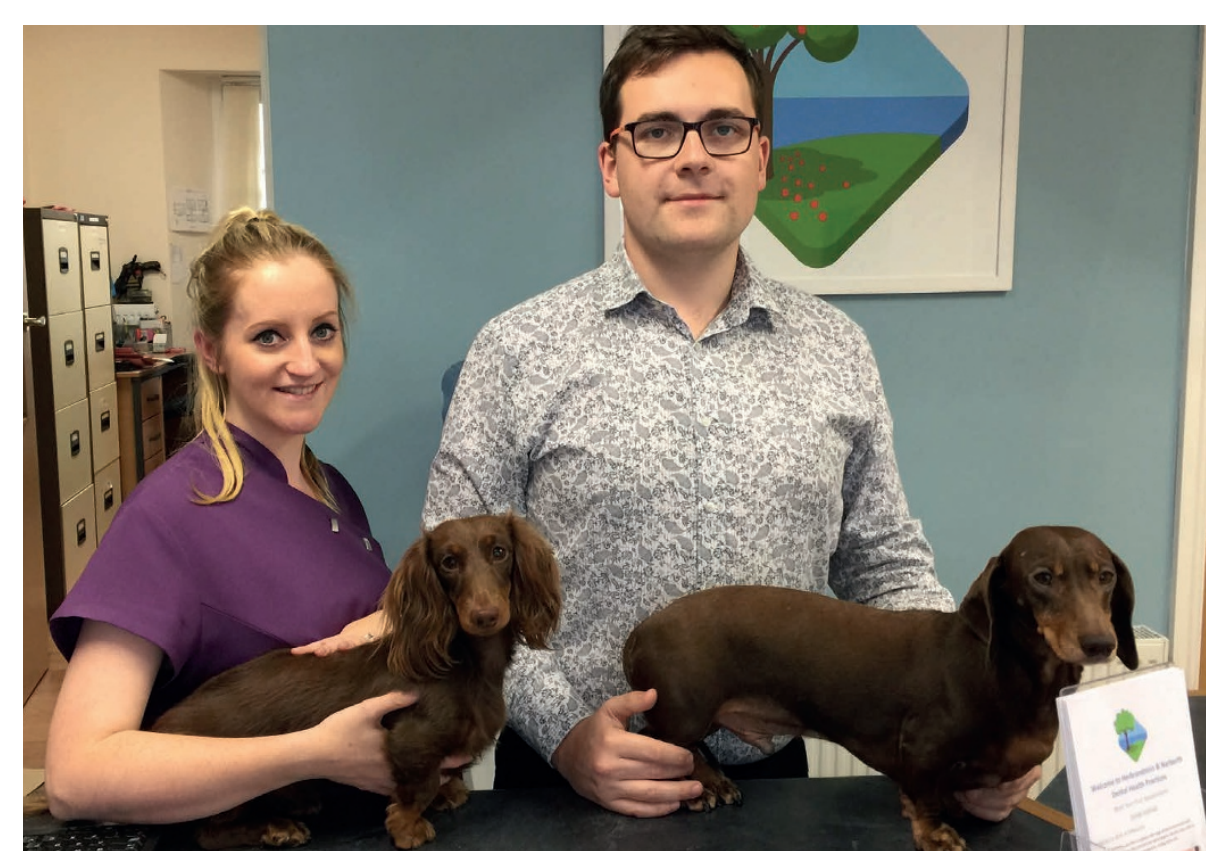

Imogen Wheeler, practice receptionist and Liam Boulcott, practice manager with Cocoa and Theo animals, a groundskeeper. We've been offered a camel which I have declined on the ground that that wouldn't be a particularly good animal to view. We have to be careful not to become a zoo because they come under regulations that are rather draconian.

'We have got very specific health and safety protocols and everything has been approved by Health Inspectorate Wales (HIW). They came in and said they couldn't find anything to find fault in.

but if you take them outside and show them the animals, then let them talk, they can open up about issues and they feel more relaxed.'

A good example cited by Boulcott is a patient who was terrified of dentists and had not been to see one for 18 years. She came to the practice and spent time with Boulcott and the animals and managed to overcome her fear.

'All the animals are incredibly tame and, effectively, I have them on the books as members of staff', he said. 'I employ somebody to look after the gardens and the 